\title{
Acute effect of low-Dye taping on dynamic plantar pressure in subjects with overpronation foot.
}

\author{
Fernando Raphael Pinto Guedes Rogério', David Jeferson dos Santos², André Felipe Silva Desiderio², \\ Eliane Regina Ferreira Sernache de Freitas ${ }^{3}$.
}

\begin{abstract}
ABSTACT
Introduction: The pronation foot is related to numerous musculoskeletal disorders, in order to reverse this biomechanical dysfunction has often been used to low-Dye taping (LDT). Objective: Evaluate the acute effect of the application of low-Dye taping (LDT) on dynamic Baropodometric parameters (DBP) in subjects with foot overpronation. Method: They evaluated 17 feet by baropodometric dynamic by midgait protocol. The pressure measurements were obtained by planting stratification of the foot region in eight shades in LDT conditions and without taping. Results: The Student $t$ test for paired samples showed a significant reduction of peak plantar pressure, mean plantar pressure, integral pressure / time largely masks studied without however generate substantial increases in other plantar regions. Conclusion: The application of LDT proved to be an efficient way to reduce the DPB in subjects with foot overpronation.
\end{abstract}

Keywords: Pronation, Pressure, Physical therapy modalites, Foot, Taping

\section{INTRODUCTION}

The foot structure is an important body segment responsible for shock absorption, transfer of forces, body propulsion and stabilization in activities of different complexities. ${ }^{(1)}$ This task is only possible due to existing biomechanical relation between the transverse arch, medial longitudinal arch (MLA) and lateral longitudinal arch. ${ }^{(2)}$

Transient changes of the MLA as subtalar and midtarsal joint pronation become fundamental in dynamic activities collaborating in loads absorption and transfer of forces during the stance phase of the gait cycle. ${ }^{(3)}$ However, when pronation becomes excessive it is increase the myofascial stress, ${ }^{(4)}$ overload in the ligamentous structures, ${ }^{(4)}$ reduction of body stability, ${ }^{(5)}$ kinematic alterations ${ }^{\left({ }^{6}\right)}$ and changes in muscle activation during gait ${ }^{(7)}$ predisposing to pain syndromes and repetitive strain injuries. ${ }^{(8)}$

Several interventions in order to reduce the deleterious effects of excessive pronation foot are routinely used in clinical practice in various health conditions. ${ }^{(8,9)}$ In this sense, the implementation of low-dye taping (LDT) has been ostensibly used as a way to reduce the unwanted effects on pronation plant through the implementation of an external supination force applied medially to the axis of the subtalar joint, (10) increasing the MLA and promoting joint symmetry and lower overhead on the muscle-joint structures.

The literature points ${ }^{(8,9,10)}$ that the application of LDT might increase the height of the AML changing foot posture of individuals with subtalar pronation and reducing symptoms associated with disorders of the lower extremities. Bill Vicenzino et al ${ }^{(11)}$ investigated the responses of application of LDT on dynamic plantar pressure and foot posture of asymptomatic individuals with feet pronation. Their findings highlighted the relevant contribution of the application of LDT on the height of the AML associated with the significant decline of the contact area of the medial region of the foot during walking and running activity.

Franettovich et al (12) investigated the effect of the continuous use of LDT application on foot posture and neuromuscular control of the lower limb during the gait cycle, the results suggest that the continued use of LDT could produce changes in foot posture without, however, cause changes in neuromuscular responses during the March in asymptomatic individuals. Lunen et al ${ }^{(13)}$ conducted a study to determine the

Corresponding author: Name: Fernando Raphael Pinto Guedes Rogério. Address: Rua Paranaguá, 1900, Edifício studio V, apto. 803. Londrina (PR), Brasil. CEP: 86015030 Telephone: +55 (43) 9926-0932 E-mail: fernandopintoguedes@hotmail.com

1. Mestrando em Ciências da Reabilitação pelo Programa de Mestrado e Doutorado em Ciências da Reabilitação (UEL/UNOPAR), Professor do curso de fisioterapiaUNOPAR, Londrina (PR), Brasil.

Full list of author information is available at the end of the article.

Financial support: There was no financial support for this study. 
effect of the LDT application on pain intensity and pressure plant by baropodometric evaluation (in-shoes) during walking and running in individuals with plantar fasciitis. Their findings have shown that the LDT exerted significant effects in reducing dynamic baropodometric parameters represented by the peak and middle plantar pressure in the region of the hindfoot and corroborating to the reduction of pain of these patients.

The effect of LDT on dynamic baropodometric parameters remain inconclusive due to heterogeneity of findings in the literature as well as methodological differences related to the equipment used to obtain the plantar pressure, adopted gait protocols and foot characteristics of the studied samples. Therefore, this research aims to verify the immediate effect of the LDT application on dynamic plantar pressure in asymptomatic individuals with foot pronation.

Our hypothesis is that the LDT application will significantly modify the immediate responses of the plantar pressures during walking in asymptomatic individuals with feet pronation.

\section{METHOD}

This is a quantitative, transversal and quasi-experimental study, conducted in the facilities of UNOPAR. The study complies with the ethical principles involving human research and the Research Ethics Committee of the local institution approved it under the protocol 0061/14. The volunteers were recruited voluntarily through verbal invitation. They were informed about the methodological procedures of the study and then they signed the free, prior and informed consent (FPIC), in accordance with resolution 196/96 of the National Health Council (CNS).

\section{Sample}

The study initially included a convenience sample of 20 healthy university students (Figure 1 ) that met the following inclusion criteria: individuals aged between 18 and 40 years; presenting pronation of the hindfoot; presenting good functionality in the lower limbs, evaluated through the Lequesne's algofunctional questionnaire for the hip joint with a score of $\leq 4$, Lysholm's knee questionnaire with a score of $\geq 84$ and Foot and Ankle Outcome Score (FAOS) with a score of $\geq 84$. In addition, do not perform moderate or vigorous physical activity 24 hours before data collection and do not drink alcohol or drugs that may influence data collection.

Were excluded individuals with a history of injuries, surgeries and fractures in lower limbs during the last 6 months, presence of cardiac and respiratory diseases, vestibular dysfunction, visual disturbances and the ones who made use of any medication that may interfere with the ability to wakefulness and balance. Such exclusion criteria were applied in the form of interview and therefore do not exclude the possibility of ignorance about such problems by the participants. Thus, the study included 11 individuals comprising a total of 17 feet evaluated.

\section{Analysis of foot posture}

To obtain the footprint the following procedure was conducted: the paint roller was initially dyed with paint on the stamp pad, then the roller plated was passed in ink on the textured surface of the foot imprinter and placed the paper of dimensions $370 \mathrm{~mm} \times 180 \mathrm{~mm}$ covered by rubber film on the flat plan of the foot imprinter. After the preparation of the equipment, the evaluated remained in bipedal support, with comfortable support base, weight symmetrically distributed among the members and arms relaxed alongside the body. Then the foot imprinter was positioned in front of the evaluated who took a step on the equipment maintaining body weight, after the transfer of weight the individual removed his foot and then the measurement of the opposite member was conducted.

The plantigraphy in which the foot was in the center of the paper sheet, the demarcation of the footprint was free of paint imperfections and the ones with no losses in the foot morphology as poor visibility of the footprint was accepted.

Through the acquisition of the plantigraphy, the specific anatomical points were determined and measured in different regions and then determined the foot posture. At first, it was measured the longitudinal extent of the foot that included the union of the most extreme points, after the calcaneus $(A)$ to the most distal toe of the foot $(B)$ being determined the $A B$ line segment. Then it was defined the foot isthmus line segment (C), through the determination of the midpoint of the $A B$ segment $(A B / 2)$ and measuring the width of this segment to the external point of the foot, corresponding to the measure midfoot. As measures to determine the width of the forefoot

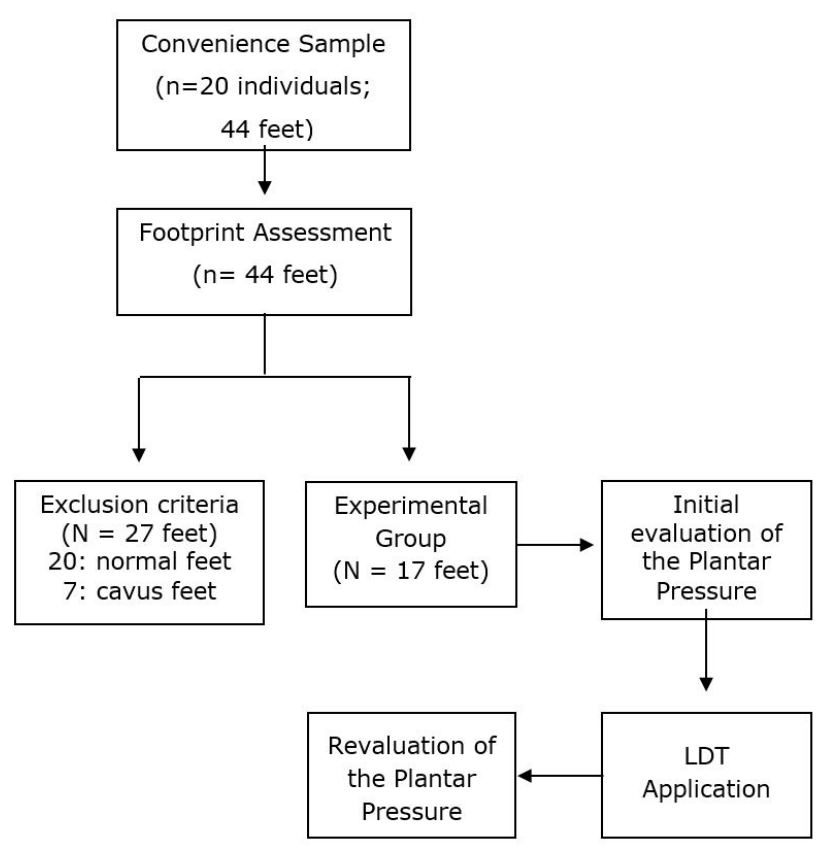

Figure 1. Sample flowchart 
was traced a surfacing line being tangential to the media and lateral edges of the forefoot and then determined the width of the forefoot (D).

Classification of foot posture by Viladot method: This method takes into consideration the relation of the midfoot with the forefoot. Thus, flat feet were considered those whose footprint presented the midfoot region (C) with a width equal or greater than half of the forefoot $(C \geq 1 / 2 D)$; cavus feet were those with a reduction in the footprint area in its middle part, less than one third of the forefoot or with disappearance $(C \leq 1 / 3 D$ or $C=0)$. Finally, the feet were classified as normal those which the midfoot measure ranged from one-half to one third of the width of the forefoot $(1 / 2 D \leq C \leq 1 / 3 D)$. ${ }^{(14)}$

\section{Reproducibility}

It was realized the reproducibility of inherent assessments of the dynamic plantar pressure (DPP) before experimental procedure. It was recruited 10 volunteers ( 4 men and 6 women) being used the same inclusion and exclusion criteria for the main study.

For DPP reproducibility, the subjects came to the laboratory for two consecutive days being conducted familiarization of the evaluated with the midgait protocol and subsequently collected three measures of DPP of the dominant side. Data collection was performed by two physiotherapists previously trained, in which one was responsible for handling the software while the other performed the instructions and observation of the rejection criteria of DPP measures.

\section{Experimental Procedure}

Initially were collected personal data, anthropometric characteristics and performed familiarization with the dynamic baropodometry protocol. During the familiarization subjects were instructed to walk freely in self-selected speed on the baropodometer during a 3 minutes period in a hallway of 10 meters with a centralized platform in the middle of the path. After familiarization, the equipment calibration was realized through the insertion of the body weight value of the evaluated on the Footwork pro 3.8.1.1 software, followed by the dynamic baropodometry pre intervention. Subsequently, the LDT application was performed and then the subjects underwent a new baropodometry.

\section{Dynamic Baropodometry}

To obtain the dynamics baropodometric parameters were used the midgait protocol. ${ }^{(15)}$ In this protocol, the individual is in quiet-standing position with parallel feet, relaxed arms and looking in the horizontal plane, then marches over a distance of 10 meters contacting only one foot on the baropodometer positioned in the center of the trajectory. The subjects walked in self-selected speed and they were instructed to walk naturally with arms alongside of the body and looking in the horizontal plane, crossing the entire trajectory until the end of the runway after contact with the platform. It was performed three valid measures in pronator foot side and the average of all tests in order to obtain the baropodometric parameters. ${ }^{(16)}$

This protocol allows a more precise analysis in relation to the gait cycle compared to abbreviated gait protocols as methods of one, two and three steps. The abbreviated gait protocols are likely to lower values of pressure and strength components while walking and therefore can generate unrepresentative results in the usual in the gait of the individual; also abbreviated protocols quantify the baropodometric parameters during the acceleration phase of the gait. ${ }^{(16,17)}$ Considering that the gait is a continuous and dynamic cycle the midgait protocol has been used as a reference protocol for determining these variables.

For the data collection, all individuals held a familiarization with the experimental protocol in which they walked for three minutes on the platform to ensure the realization of a comfortable and habitual gait. During the data collection procedure, subjects were encouraged to adopt a natural gait pattern walking at a self-selected speed.

Were rejected the dynamic plantar pressure in which the foot didn't touch the platform, changes in gait pattern to ensure contact with the platform, purposeful gait abnormalities, adjust the step prior to reaching the platform, occurrence of imbalances during operation, beginning out of the pre stipulated marking, pause on the equipment or do not complete the final steps after contact with the equipment.

\section{Equipment}

For data collection of DPP were utilized the pressure plataform of Footwork Pro model, AM3 France, with active surface of $490 \mathrm{~mm} \times 490 \mathrm{~mm}$, thickness of $4 \mathrm{~mm}, 4096$ calibrated capacitive sensors, captors of $7.62 \times 7.62 \mathrm{~mm}$, frequency of data collection of $200 \mathrm{~Hz}$ and captor maximum pressure of $120 \mathrm{~N} / \mathrm{cm}^{2}$.

\section{Variables analyzed by the dynamic baropodometry}

The measures related to DPP analysis obtained pre and post intervention were peak plantar pressure (PPP), mean plantar pressure (MPP) and plantar pressure time integral (PPTI). These variables were chosen due to their clinical applicability in the functional aspect of disorders associated with the feet, risk of ulcerations ${ }^{(18)}$ and measures of effectiveness to therapeutic interventions. $^{(19,20)}$

These variables were determined in 8-foot regions through AutoMask function of FootWork Pro 2.9.1 software. The following regions were analyzed (figure 2): internal calcaneal region (ICR), external calcaneal region (ECR), midfoot (MF), 1을 metatarsus (1MT), 2ㅇ and 3 metatarsus (2/3 MT), $4^{\circ}$ and $5^{\circ}$ metatarsus $(4 / 5 \mathrm{MT})$, hallux $(\mathrm{H})$ and other fingers (OF). These regions were chosen aiming to provide detailed information about the function of different plantar areas during the gait and they were analyzed in previous studies. ${ }^{(32)}$ 


\section{Application of the low-dye taping}

For LDT application, was used a LeukoTape Sportstape rigid tape (Beirsdorf, AG, Hamburg, Germany). Initially was conducted the cleaning of the foot with water and ethanol and then was realized the procedure as described by Vicenzino. (21) Therefore, were applied a tape from the first metatarsal head covering the medial side of the foot, around the posterior region of the calcaneus and being fixed to the fifth metatarsal head. Sequentially were applied transverse strips starting from the side region of the foot covering the plantar surface and anchored to the medial region of the foot. Were continuously applied these strips distally to proximally so that the subsequent strip would cover half the width of the previous strip. They were applied all over the medial surface of the foot.

\section{Statistical analysis}

The statistical analysis were realized using the SPSS program 20.0 version to Windows (SPSS Inc, Chicago, EUA). Before inferential analysis, all the data were tested for normality using the Kolmogorov-Smirnoff test. All variables showed normal distribution and thus was used parametric statistics for data interpretation. To obtain the dynamic baropodometric values were used the average of three tests and for that was made the sum of all the values and the division by the number of trials. (34) For the analysis of reproducibility between the dynamic baropodometric evaluations, were calculated the Intraclass Correlation Coefficient (ICC) with its confidence interval established in 95\% (CI 95\%). The interpretation of ICC was conducted in accordance with Portney and Watkins ${ }^{(22)}$ in which the values higher than 0.75 indicate good reliability, the values ranging $0.5-0.75$ indicate moderate reliability and the values lower than 0.5 indicate low reliability. For comparison of the LDT application effect on the plantar pressure parameters was performed Student's t-test for paired samples. Were calculated the effect size by the estimate of Cohen's $d^{(23)}$ in which were considered little effect if $(0.20<d<0.50)$, moderate effect if $(0.50<d<0.80)$ and great effects if $(d>0.80) .{ }^{(38)}$

\section{RESULTS}

\section{Characteristics of the participants}

The sample consisted of 11 healthy young volunteers ( 5 male and 6 female), mean age ( $23.18 \pm 1.32$ years), weight $(66.43 \pm 16.03 \mathrm{~kg})$, height (171 \pm 10.86$)$, body mass index $\left(23.72 \pm 1.55 \mathrm{~kg} / \mathrm{cm}^{2}\right)$ and footwear number (39.09 \pm 3.30$)$.

\section{Reproducibility between the dynamic baropodometric evaluations}

The reproducibility between the two-day trial represented by the ICC as well as its $\mathrm{Cl} 95 \%$ are shown in Table 1. Regarding PPTI, variables were found with moderate to high reproducibility not being found with low reproducibility

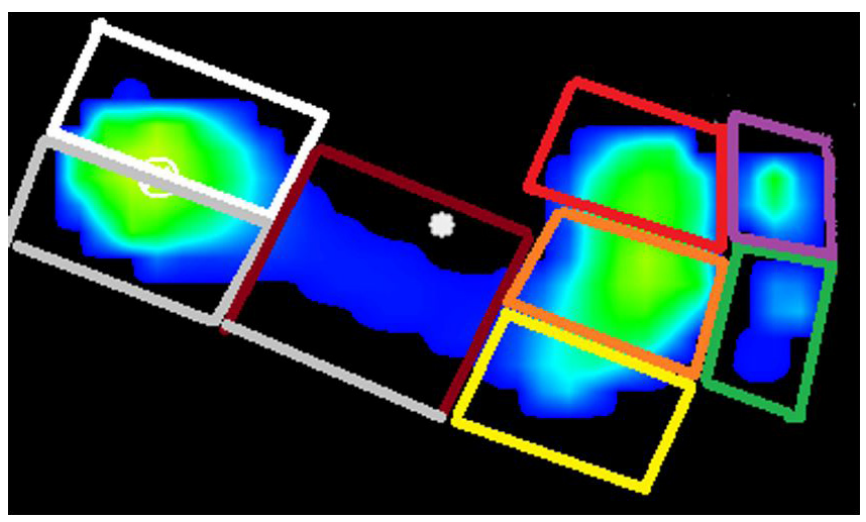

Figure 2. Example of footprint obtained by Footwork Pro ${ }^{\circledR}$ system (AM Cube, France), reproduction of the seven regions in which we determined footprint masks for analysis of dynamic baropodometric parameters.

among the evaluated masks. The masks that showed high reproducibility were $\mathrm{M} 1$ [0.88 (0.70-0.95)], M4/M5 [0.82 (0.54-0.93)], midfoot [0.95 (0.87-0.98)], external calcaneal region $[0.75(0.35-0.90)]$.

Regarding MPP, just hallux [0.87(0.67-0.95)], M2/M3 [0.84 (0.59-0.93)], midfoot [0.85 (0.63-0.94)] and internal calcaneal region [0.84(0.58-0.93)] showed high reproducibility. Furthermore, the variables with higher reproducibility in relation to PPM were M1 [0.87 (0.67-0.95)], M2/M3 [0.90 (0.76-0.94)], M4/M5 [0.89 (0.73-0.96)], midfoot [0.90 (0.75-0.96] and internal calcaneal region [0.90 (0.75-0.94)].

\section{Analysis of dynamic baropodometric parameters in pre and post LDT application}

The analysis related to the comparison between the averages of PPP ( $\mathrm{kPa}$ ) are in Table 2 . Statistical differences in pre and post LDT application were found between the averages of the following masks: hallux (387.47 \pm 60.11 ; $355.29 \pm 75.31$, $\mathrm{p}=0.13), \mathrm{M} 4 / \mathrm{M} 5$ (332.88 $\pm 88.21 ; 309.88 \pm 75.04 ; \mathrm{p}=0.04)$, midfoot $(84.23 \pm 83.31 ; 62.23 \pm 38.34 ; p=0.03)$, internal calcaneal region $(435.41 \pm 43.90 ; 412.35 \pm 33.28 ; p=0.02)$ and external calcaneal region $(414.41 \pm 44.01 ; 390.94 \pm 38.74$; $p=0.00)$. Were found moderate effect in the following regions: M4/M5 ( $d=0.53)$, midfoot $(0.56)$, external calcaneal ( $d=0.61)$ and internal calcaneal (0.52).

Table 3 shows the comparison between the averages of MPP ( $\mathrm{kPa}$ ) and their masks. Statistical differences were found just between the following masks: M2/M3 (252.11 \pm 32.95 ; $235.82 \pm 39.84 ; p=0.04)$, midfoot $(32.58 \pm 22.17 ; 49.05 \pm 22.69$; $\mathrm{p}=0.01$ ) and internal calcaneal region (390.94 \pm 38.74 ; $228.88 \pm 19.98, p=0.01)$. Moderate effects were found in the following regions: $M 2 / M 3(d=0.53)$, midfoot $(d=0.71)$ and internal calcaneal ( $d=0.58)$.

Table 4 shows the comparison of the averages of PPTI variable (\% kPa.s $\left(\mathrm{cm}^{2}\right)$ and their masks. Statistical difference was detected as well as moderate effect just in the midfoot mask (7.06 $\pm 6.39 ; 4.65 \pm 3.93 ; p=0.01 ; d=0.71)$. 


\section{Adverse effects}

There were no adverse effects such as skin irritation, hypersensitivity or dermatological lesions associated with the use of LDT.

\section{DISCUSSION}

This research showed that the LDT application reduced significantly dynamic baropodometric parameters represented by PPP, MPP and PPTI in the following regions: metatarsus $(1$ 으, 2으, 3으, 4음 and 5ㅇ) , midfoot and hindfoot in young individuals with flat feet diagnosed by plantigraphy. The effect of LDT application occurred more pronounced in the PPP, MPP and PPTI variables, respectively. Our results corroborate partially with previous studies that verified a reduction of plantar pressure in the forefoot and midfoot $^{(24)}$ after application of this technique.

The electronic baropodometry platform method, used in this study to quantify plantar pressures, provides specific quantitative data of DPP established through the interaction of the foot structure with the ground. Its analysis allows to evaluate the interaction in dynamic activities such as gait and running as well as to evaluate the effect of specific interventions such as the use of bandages and orthoses. However, while this interaction can be established, the alterations found in plantar pressures do not imply directly in biomechanical changes in foot structure, making it impossible to draw conclusions about the effect of LDT on foot arthrokinematics.

Thus, the findings of this study will be discussed based on clinical interpretations associated with the changes in PP as well as in the findings of previous investigations.

Although the changes in foot arthrokinematics cannot be evaluated by dynamic baropodometry, the study of plantar pressures provides relevant information on the foot behavior and its relation to the load distribution in different phases of the gait cycle. Its applicability can be evidenced in the study of foot disorders, diabetic ulcers, sports medicine and rehabilitation. ${ }^{(25)}$

This research provides important information on the pressor responses underlying the LDT application. Previous studies such as Russo and Chipchase ${ }^{(26)}$ evaluated the effect of LDT application on baropodometric parameters represented by PPP in healthy subjects with normal foot posture assessed by plantigraphy. The authors found a significant increase in PPP in masks corresponding to the external calcaneal, internal calcaneal and lateral midfoot regions associated with a reduction of the PPP in the medial region of the midfoot.

Our findings corroborate partially with these authors, as detected a significant reduction in the masks corresponding to

Table 1. Reproducibility between the dynamic baropodometric evaluations (Intraclass Correlation Coefficient [ICC]) to obtain the baropodometric parameters in each foot mask.

\begin{tabular}{lccc}
\hline \multicolumn{1}{c}{ Masks } & PPTI ICC (95\%Cl) & PPP ICC (95\%Cl) & MPP ICC (95\%CI) \\
\hline Hallux & $0.73(0.18-081)$ & $0.87(0.67-0.95)$ & $0.73(0.32-0.90)$ \\
Other Fingers & $0.61(0.10-0.85)$ & $0.61(0.10-0.85)$ & $0.20(0.69-1.05)$ \\
M1 & $0.88(0.70-0.95)$ & $0.54(0.42-0.78)$ & $0.87(0.67-0.95)$ \\
M2\M3 & $0.67(0.16-0.87)$ & $0.84(0.59-0.93)$ & $0.90(0.76-0.94)$ \\
M4\M5 & $0.82(0.54-0.93)$ & $0.66(0.11-0.86)$ & $0.89(0.73-0.96)$ \\
Midfoot & $0.95(0.87-0.98)$ & $0.85(0.63-0.94)$ & $0.90(0.75-0.96)$ \\
External calcaneal & $0.75(0.35-0.90)$ & $0.73(0.30-0.89)$ & $0.70(0.21-0.88)$ \\
Internal calcaneal & $0.66(0.10-0.86)$ & $0.84(0.58-0.93)$ & $0.90(0.75-0.94)$ \\
\hline
\end{tabular}

PPTI: plantar pressure time integral (\% kPa.s $\left(\mathrm{cm}^{2}\right)$; PPP: peak plantar pressure (KPa); MPP: mean plantar pressure (KPA); ICC: Intraclass Correlation Coefficient; $95 \% \mathrm{Cl}$ : Confidence interval.

Table 2. Comparison between the averages of PPP and effect size in pre and post LDT application.

\begin{tabular}{|c|c|c|c|c|}
\hline Foot Masks & $\begin{array}{l}\text { PPP (KPa) } \\
\text { Pre Post }\end{array}$ & $\begin{array}{l}\text { Difference between the } \\
\text { averages }\end{array}$ & Effect Size & Value of $p$ \\
\hline Hallux & $387.47 \pm 355.29 \pm 60.1175 .31$ & 32.17 & 0.38 & $0.03^{*}$ \\
\hline Other Fingers & $335.58 \pm 297.47 \pm 78.25108 .43$ & 38.11 & 0.34 & 0.17 \\
\hline M1 & $334.52 \pm 333.29 \pm 68.6366 .77$ & 1.23 & 0.02 & 0.93 \\
\hline $\mathrm{M} 2 \backslash \mathrm{M} 3$ & $392.17 \pm 380.11 \pm 46.5648 .11$ & 12.05 & 0.33 & 0.18 \\
\hline M4\M5 & $332.88 \pm 309.88 \pm 88.2175 .04$ & 23.00 & 0.53 & $0.04 *$ \\
\hline Midfoot & $84.23 \pm 62.23 \pm 83.3138 .34$ & 22.00 & 0.56 & $0.03^{*}$ \\
\hline External calcaneal & $435.41 \pm 412.35 \pm 49.9033 .28$ & 23.05 & 0.61 & $0.02 *$ \\
\hline Internal calcaneal & $414.41 \pm 390.94 \pm 44.0138 .74$ & 23.47 & 0.52 & $0.01 *$ \\
\hline
\end{tabular}

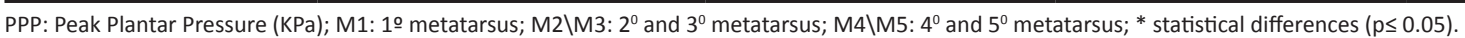


Table 3. Comparison between the averages of MPP and effect size in pre and post LDT application.

\begin{tabular}{lcccc}
\hline \multicolumn{1}{c}{ Foot Masks } & \multicolumn{1}{c}{$\begin{array}{c}\text { MPP }(\mathrm{KPa}) \\
\text { Pre Post }\end{array}$} & $\begin{array}{c}\text { Difference between the } \\
\text { averages }\end{array}$ & Effect Size & Value of $\mathbf{p}$ \\
\hline Hallux & $201.11 \pm 192.11 \pm 47.7244 .79$ & 9.00 & 0.17 & 0.47 \\
Other Fingers & $116.58 \pm 111.17 \pm 35.9845 .67$ & 5.41 & 0.14 & 0.55 \\
M1 & $212.05 \pm 211.23 \pm 71.3660 .10$ & 0.82 & 0.01 & 0.94 \\
M2 $\backslash$ M3 & $252.11 \pm 235.82 \pm 32.9539 .34$ & 16.29 & 0.53 & $0.04 *$ \\
M4\M5 & $150.82 \pm 146.70 \pm 54.8554 .50$ & 4.11 & 0.11 & 0.64 \\
Midfoot & $49.05 \pm 32.58 \pm 22.6922 .17$ & 16.47 & 0.79 & $0.01^{*}$ \\
External calcaneal & $239.52 \pm 228.35 \pm 34.9122 .31$ & 11.17 & 0.43 & 0.09 \\
Internal calcaneal & $390.94 \pm 228.88 \pm 38.7419 .98$ & 162.05 & 0.58 & $0.01^{*}$ \\
\hline
\end{tabular}

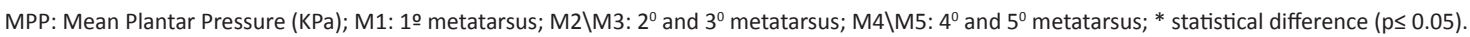

Table 4. Comparison between the averages and effect size of PPTI in pre and post LDT application.

\begin{tabular}{|c|c|c|c|c|}
\hline Foot Masks & $\begin{array}{l}\text { PPTI (KPa) } \\
\text { Pre Post }\end{array}$ & $\begin{array}{c}\text { Difference between the } \\
\text { averages }\end{array}$ & Effect Size & Value of $p$ \\
\hline Hallux & $26.94 \pm 26.94 \pm 10.7410 .85$ & 0.00 & 0.00 & 1.00 \\
\hline Other Fingers & $14.12 \pm 13.94 \pm 6.679 .18$ & 0.17 & 0.02 & 0.92 \\
\hline M1 & $43.53 \pm 41.24 \pm 16.3714 .29$ & 2.29 & 0.20 & 0.40 \\
\hline $\mathrm{M} 2 \backslash \mathrm{M} 3$ & $56.88 \pm 53.29 \pm 6.319 .10$ & 3.58 & 0.45 & 0.07 \\
\hline M4\M5 & $28.29 \pm 27.18 \pm 8.309 .54$ & 1.11 & 0.14 & 0.56 \\
\hline Midfoot & $7.06 \pm 4.65 \pm 6.393 .93$ & 2.41 & 0.71 & $0.01^{*}$ \\
\hline External calcaneal & $31.12 \pm 30.35 \pm 8.116 .16$ & 0.76 & 0.13 & 0.15 \\
\hline Internal calcaneal & $39.53 \pm 37.41 \pm 9.6011 .56$ & 2.11 & 0.36 & 0.58 \\
\hline
\end{tabular}

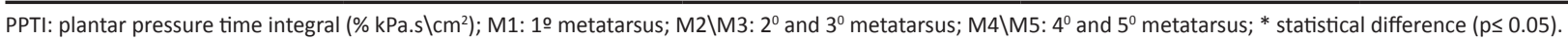

the midfoot, internal / external calcaneus and fourth and fifth metatarsus without, however, result in substantial increases in other foot areas. Concomitantly, our study showed an effect size more pronounced on the midfoot region showing a clinical improvement in the reduction of PPP in this area which receives high loads during the gait cycle due to reduction of the MLA.

Besides the already exposed, this study ${ }^{(26)}$ besides the already exposed, this study ${ }^{(26)}$ conducted its intervention in individuals with normal foot posture which can cause a behavior change in relation to the responses of LDT application when compared with individuals with flat feet.

Moreover, Lange et al (27) studied the acute responses of PPP, MPP and baropodometric parameters induced by the LDT application in individuals with foot pronation evaluated by the navicular drop test. The authors demonstrated an increase of MPP in the lateral midfoot as well as a reduction of PPP in external and internal calcaneal region and central and medial forefoot.

Although this study showed similar results to our findings, the methodological differences end up making the results difficult to compare, firstly, the method used for the diagnosis of foot posture, navicular drop test and plantigraphy. In this sense, Menz and Munteanu ${ }^{(28)}$ evaluated the association between three clinical measures to obtain the foot posture (plantar arch index, the foot posture index and navicular height), with the gold standard method for this diagnosis, angular measurements obtained by radiographs (navicular height, tilt angle of the calcaneus and calcaneal angle with first metatarsus) in 95 elderly. Their findings showed a strong correlation between the three clinical measures analyzed with the x-rays measurements. Although this study shows a good concurrent validity between the methods, it used as samples elderly which eventually reduce the external validity when evaluating young healthy subjects.

Secondly, the study of Lange et al ${ }^{(27)}$ used ten foot masks to evaluated the LDT effect on the dynamic baropodometry parameters while our study used only eight anatomical regions. Thus, the smaller number of masks used in our study may have underestimated the effect of the LDT application on foot pressure parameters making it impossible to detect major changes in different foot areas. So we can justify the non-appearance of pressor increases in certain masks as the lateral midfoot found in the above studies. 
Our study showed a significant reduction of the PPP and MPP in masks of internal and external calcaneal regions through LDT application. This effect happens due to LDT change the resultant of the forces acting on the calcaneus area, promoting a joint restriction of subtalar and midtarsal pronation, favoring joint symmetry with subsequent greater efficiency in the distribution of plantar loads during gait. Although the PP has been reduced in this area with moderate effect size, significant increase was not found in PP parameters in the foot medial region as shown in the literature. (27)

The significant reduction of PPP, MPP and PPTI may have been triggered because the direction of application of the LDT forces which seeks to create a vector of supinator force in this segment associated with a facilitated muscle activity of the tibialis posterior muscle. The clinical relevance of this effect is in the fact that the reduction of PP in the midfoot tends to indicate greater stabilization of the MLA during the response period of the load in the stance phase of the gait cycle, demonstrating that the LDT application was able to change the foot posture.

Therefore, can be infer that the LDT significantly reduced these parameters with moderate effect size, however, the duration of the effect as the clinical potential of these changes in various clinical conditions remains not established in the literature.

Among the limitations of this study, we evidence the short evaluation period after the LDT application. We investigated the immediate effect of this application in previous studies ${ }^{(29,30)}$, however, this research has not been able to evidence the PP behavior over a significant period of time so requiring new studies that seek to investigate this topic. In addition, the reduced sample size ends up involving the internal and external validity, so these findings should be interpreted with caution.

Although these limitations have been found, this study provides crucial information about the behavior of the PP in healthy subjects with flat feet which eventually contribute to the understanding of the mechanisms which involves the redistribution of plantar pressure and thus allow new research in subjects with foot pathologies.

\section{CONCLUSION}

The LDT application showed to alter significantly the plantar pressure values during gait in healthy young subjects. Its application significantly reduced dynamic baropodometry parameters of calcaneus and midfoot regions without causing increase in the pressure in other plantar regions and redistributing plantar pressure during walking.

\section{AUTHOR'S CONTRIBUTIONS}

FRPGR is responsible for the conception, design of the study, analysis and interpretation of the data and drafting the article; DJS and AFD were responsible for data collection; ERFSF is responsible for revised the manuscript critically. All authors approved the final version of the manuscript.

\section{CONFLICTS OF INTEREST}

The authors declare that they have no conflicts of interest in the research.

\section{AUTHOR DETAILS}

${ }^{2}$ Discente do curso de Fisioterapia (UNOPAR), Londrina (PR), Brasil. ${ }^{3}$ Doutora em Ciências pela Universidade Federal de São Paulo/Escola Paulista de Medicina (UNIFESP/EPM). Docente do Programa de Mestrado e Doutorado em Ciências da Reabilitação (UEL/UNOPAR). Docente do curso de Fisioterapia (UNOPAR), Londrina (PR), Brasil.

\section{REFERENCES}

1. Saltzman CL, Nawoczenski DA. Complexities of foot architecture as a base of support. Journal of Orthopaedic and Sports Physical Therapy 1995;21:354-360.

2. Murphy DF, Connolly DA, Beynnon BD. Risk factors for lower extremity injury: a review of the literature. Br J Sports Med. 2003;37:13-29.

3. Razeghi M, Batt M. Biomechanical analysis of the effect of orthotic shoe inserts. A review of the literature. Sports Medicine 2000;29:425-438.

4. Hintermann B, Nigg BM. Pronation in Runners: Implications for injuries. Sports Med 1998;26(3):169-176.

5. Williams DS, McClay IS, Hamill J. Arch structure and injury patterns in runners. Clinical Biomechanics 2001;16:341-347.

6. Mundermann A, Nigg BM, Humble RN, Stefa- nyshyn DJ. Foot orthotics affect lower extremity kinematics and kinetics during running. Clin Biomech (Bristol, Avon). 2003;18:254-262.

7. Nigg BM. The role of impact forces and foot pronation: a new paradigm. Clin J Sport Med. 2001;11: 2-9.

8. Radford JA, Landorf KB, Buchbinder R, Cook C. Effectiveness of low-Dye taping for the short-term treatment of plantar heel pain: a randomised trial. BMC Musculo- skelet Disord. 2006;7:64. http://dx.doi. org/10.1186/1471-2474-7-64.

9. O'Sullivan K, Kennedy N, O'Neill E, Ni Mhainin U. The effect of low-dye taping on rearfoot motion and plantar pressure during the stance phase of gait. BMC Musculoskelet Disord. 2008;9:111.

10. Del Rossi G, Fiolkowski P, Morodyski MB, Bishop M, Trimble M. For how long do temporary techniques maintain the height of the longitudinal arch? Phys Ther Sport 2004;5:84-89.

11. Vicenzino B, McPoil T, Buckland S. Plantar foot pressures after the augmented low dye taping technique. J Athl Train. 2007;42(3):374-80.

12. Franettovich $M$, Chapman A, Blanch $P$, Vicenzino B. Continual use of augmented low-Dye taping increases arch height in standing but does not influence neuromotor control of gait. Gait Posture. 2010;31(2):247-50.

13. Van Lunen B, Cortes N, Andrus T, Walker M, Pasquale M, Onate J. Immediate effects of a heel-pain orthosis and an augmented low-dye taping on plantar pressures and pain in subjects with plantar fasciitis. Clin J Sport Med. 2011 Nov;21(6):474-9.

14. Volpon JB. Footprint analysis during the growth period. J pediatr Orthop 1994;14:83-5.

15. Morlock M, Mittlmeiser T. First step method versus full gait method: results for comparison. Eur J Phys Rehab Med 1992;2:S33.

16. Hughes J, Pratt I, Linge K, Clark P, Kienerman L. Reliability of pressure measurements: the EMED-F system. Clin Biomech 1991;6:14-18.

17. Wearing S, Urry S, Smeathers J, Battistutta D. A comparison of gait initiation and termination methods for obtaining plantar foot pressures. Gait Posture 1999;10:255-263.

18. Barn R, Waaijman R, Nollet F, Woodburn J, Bus SA. Predictors of barefoot plantar pressure during walking in patients with diabetes, peripheral neuropathy and a history of ulceration. PLoS One. 2015;3:10(2):e0117443. doi: 10.1371/journal.pone.0117443. eCollection 2015. PubMed PMID: 25647421; PubMed Central PMCID: PMC4315609. 
19. Chakraborty PP, Ray S, Biswas D, Baidya A, Bhattacharjee R, Mukhopadhyay $P$, et al. A comparative study between total contact cast and pressurerelieving ankle foot orthosis in diabetic neuropathic foot ulcers. J Diabetes Sci Technol. 2015 Mar;9(2):302-8.

20. Maddali Bongi S, Del Rosso A, Mikhaylova S, Landi G, Ferretti B, Cavigli $E$, et al. A comparison of two podiatric protocols for metatarsalgia in patients with rheumatoid arthritis and osteoarthritis. Clin Exp Rheumatol. 2014 Nov-Dec;32(6):855-63.

21. Vicenzino B, Feilding J, Howard R, Moore R, Smith S. An investigation of the anti-pronation effect of two taping methods after application and exercise. Gait Posture. 1997;5:1-5.

22. Portney L, Watkins M. Foundations of clinical research. Application to practice. 2 edition. London: Prentice Hall Ltd; 2000.

23. Cohen J. Statistical Power Analysis for the Behavioral Sciences (2nd Edition). Hillsdale, NJ: Lawrence Earlbaum Associates; 1988.

24. Russo SJ, Chipchase LS. The effect of low-Dye taping on peak plantar pressures of normal feet during gait. Aust J Physiother. 2001;47:239-244.
25. Deschamps K, Staes F, Desmet D, Roosen P, Matricali GA, Keijsers N, et al. A color-code based method for the interpretation of plantar pressure measurements in clinical gait analysis. Gait Posture. 2014 Dec 22. pii: S0966-6362 (14)00796-6. doi: 10.1016/j.gaitpost.2014.12.013. [Epub ahead of print]

26. Russo SJ, Chipchase LS. The effect of low-Dye taping on peak plantar pressures of normal feet during gait. Aust J Physiother. 2001;47:239-244.

27. Lange B, Chipchase L, Evans A. The effect of low-Dye taping on plantar pressures, during gait, in subjects with navicular drop exceeding $10 \mathrm{~mm}$. J Orthop Sports Phys Ther. 2004 Apr;34(4):201-9. PubMed PMID: 15128190.

28. Menz HB, Munteanu SE. Validity of 3 clinical techniques for the measurement of static foot posture in older people. J Orthop Sports Phys Ther 2006;36:179.

29. Vicenzino B, Feilding J, Howard R, Moore R, Smith S. An investigation of the anti-pronation effect of two taping methods after application and exercise. Gait Posture. 1997;5:1-5.

30. Hadley A, Griffiths S, Griffiths L, Vicenzino B. Antipronation taping and temporary orthoses. Effects on tibial rotation and rearfoot position after exercise. J Am Podiatr Med Assoc. 1999;89:118-123. 